\title{
Two sides of a molecular surface for analysis of non-covalent interactions
}

\author{
A. V. Vologzhanina, A. A. Korlyukov \\ A.N. Nesmeyanov Institute of Organoelement Compounds RAS, 28 Vavilova str., 119991 Moscow, Russian Federation \\ vologzhanina@mail.ru
}

The identification of differences and similarities in non-covalent interactions of molecules in closely related solids (polymorphs, solvates, homologues, isostructural series, and others) is crucial for crystal engineering. However, crystalline environment also affects molecular reactivity within a "reaction cavity" or conformations of flexible molecules. Analysis of contributions of various types of non-covalent interactions to the molecular surface allows their comparison in different solid forms both in terms of molecular functional groups, and in the context of crystal field effect. The advantages of this approach include rapid calculations, consideration of 3D screening effect, and investigation of strong and week, hydrophobic and hydrophilic, rare and abundant interactions from unified positions. Particularly, the molecular Voronoi surfaces give qualitative, quantitative and visual representation of all types of intra- and intermolecular non-covalent interactions in crystals of inorganic, organic and macromolecular compounds (Fig. 1).

We applied the molecular Voronoi surfaces for analysis of non-covalent interactions in a number of bulky organic and organoelement molecular compounds. An approach to analyze conformation polymorphs was demonstrated on the example of photochromic Nsalicylideneanilines, and (2',4'-dinitrobenzyl)pyridine derivatives. Interplay of hydrogen and halogen bonds was studied for polymorphs of alkylboron-capped iron(II) and cobalt(II) hexachloroclathrochelates, and for a series of polybromide salts. The effect of crystalline environment on molecular conformations of a flexible imatinib molecule, and on a photoinitiated solid-state reaction of eicosaborate isomerization were studied. Non-covalent interactions of imatinib, abirateron and bicalutamide in their polymorphs, solvates, salts and ligand-receptor complexes were compared. The molecular Voronoi surfaces were proved to be suitable for understanding of the interplay between intermolecular strong and weak interactions, effect of a particular contact on molecular and material properties, and were found to be applicable to a large number of objects.
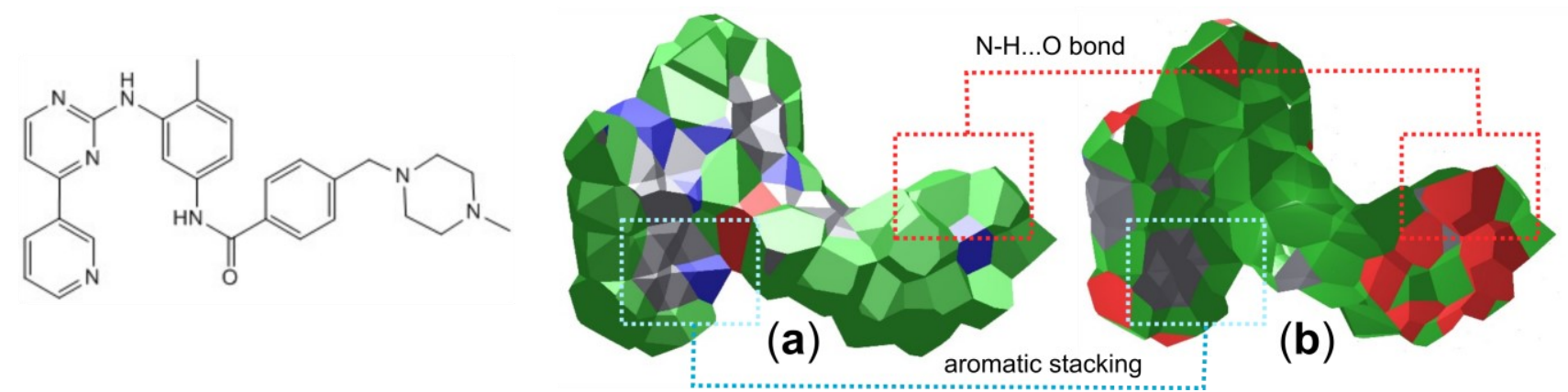

Figure 1. Molecular Voronoi polyhedron of imatinib in complex with the quinone oxidoreductase NQO2 (PDB code 3FW1) colored in accord with the nature of (a) inner, imatinib and (b) outer, receptor atoms. Color code: C-gray, H-green, N-blue, O-red. The aromatic stacking in the region of pyrid-3-yl and the $\mathrm{O} \ldots \mathrm{H}$ interactions in the region of piperidine rings are additionally marked.

Keywords: aromatic stacking; H-bonds; halogen interactions; molecular conformation; molecular Voronoi surface AVV acknowledges support of the Russian Science Foundation (project 20-13-00241). 